\title{
MESON PHYSICS AT HERA
}

\author{
JAN FIGIEL \\ The Henryk Niewodniczański Institute of Nuclear Physics Polish Academy of Sciences, \\ ul. Radzikowskiego 152, 31-342 Kraków, Poland \\ Jan.Figiel@ifj.edu.pl \\ FOR THE H1 AND ZEUS COLLABORATIONS
}

\begin{abstract}
The recent, selected results on meson production at HERA electron-proton collider from the $\mathrm{H} 1$ and ZEUS experiments are presented and interpreted within the framework of quantum chromodynamics.
\end{abstract}

Keywords: Mesons; HERA; quantum chromodynamics.

PACS numbers: 11.25.Hf, $123.1 \mathrm{~K}$

\section{Hadron Production in ep Interactions}

At high energy (as at HERA collider) the ep interaction is in fact an interaction of the virtual photon emitted from the electron(positron) with the incident proton. Such process is usually thought to proceed in two steps: interaction of the virtual photon with the partons in the proton and then fragmentation (hadronisation) of the intermediate partonic state into final hadrons, most of them being mesons. When the spatial configuration of the intermediate partonic state is small the former interaction is "hard" which implies possibility of the interpretation within the perturbative quantum chromodynamics (pQCD) whereas the latter one is typical "soft" hadronic process, usually described by the phenomenological models.

\section{Exclusive Vector Meson Production}

At HERA kinematics the diffractive coherence condition is fulfilled at low Bjorken $x$ and diffraction contributes significantly to quasi-photoproduction $\left(Q^{2} \simeq 0\right)$ but also to deep inelastic scattering (DIS), at large photon virtuality $Q^{2}$. If the virtual photon fluctuates into vector meson (VM) or recombines into real photon (deeply virtual Compton scattering - DVCS) and proton remains intact or dissociates into low-mass nucleon state one calls these processes exclusive diffraction. The "elastic" VM production (proton remains intact) is quite similar to elastic hadron-hadron interaction and can be interpretted in the framework of the Regge model, via exchange of the Pomeron trajectory. In this case typical features of the "soft" processes 
are expected, namely weak energy dependence and steep, exponential fall-off with squared four-momentum transfer $t$, of the cross section. However if $Q^{2}$ or VM mass are large the process becomes "hard" and can be described within perturbative QCD via exchange of 2 gluons or gluon ladder. In this case the cross section is proportional to squared gluon density at small $x$ which translates into its strong energy dependence. Additionally, small transverse size of the $q \bar{q}$ pair results in flatter $t$-dependence of the cross section. VM production at HERA is a testbed of QCD scales and allows observation of the transition between soft and hard regime.

Both experiments at HERA presented recently new, large statistics results on VM production. ${ }^{1-3}$ In particular the ZEUS experiment studied phtoproduction of the $\Upsilon$ meson through its decay into muon pairs. ${ }^{3}$ Because of large $\Upsilon$ mass this process is expected to be hard and this is really the case. As can be seen from Fig. 1 the cross section for $\Upsilon$ photoproduction rises strongly with increasing energy $W$ : fit to the formula $\sigma \sim W^{\delta}$ yields power $\delta \approx 1.2$ and this rise is reproduced by the QCD models.

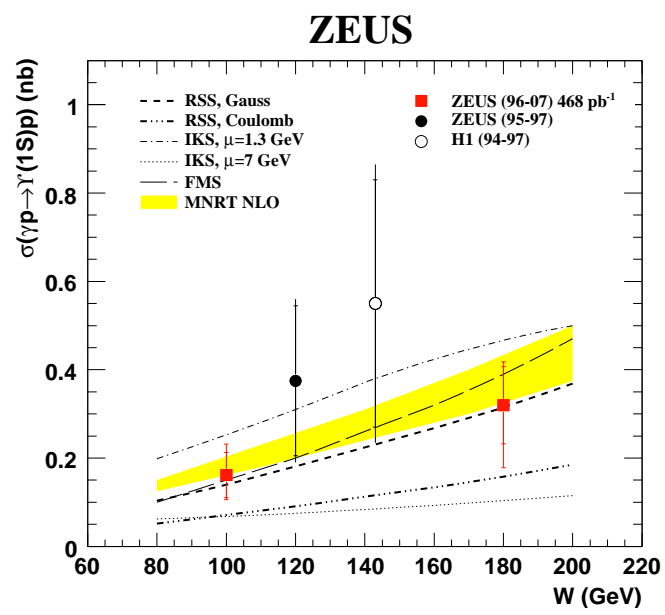

Fig. 1. The energy dependence of the cross section for the $\gamma p \rightarrow \Upsilon(1 S) p$ process ( $W$ denotes the centre of mass energy of the final hadrons). The lines and shaded area represent predictions of the various $\mathrm{pQCD}$ models.

In Fig. 2 the compilations ${ }^{1}$ of the power $\delta$ and slope $b(d \sigma / d t \sim \exp (-b t))$ for DVCS and various VMs are presented in function of the combined scale $Q^{2}+M_{V M}^{2}$. For VM the universal trends can be observed: the larger combined scale the stronger energy dependence (larger $\delta$ ) and weaker $t$ dependence (smaller slope $b$ ). This behaviour reflects transition from soft (hadron-like) scattering at low $Q^{2}$ and/or $M_{V M}^{2}$ to hard interaction at larger value of the scale, in accord with expectations from QCD. 

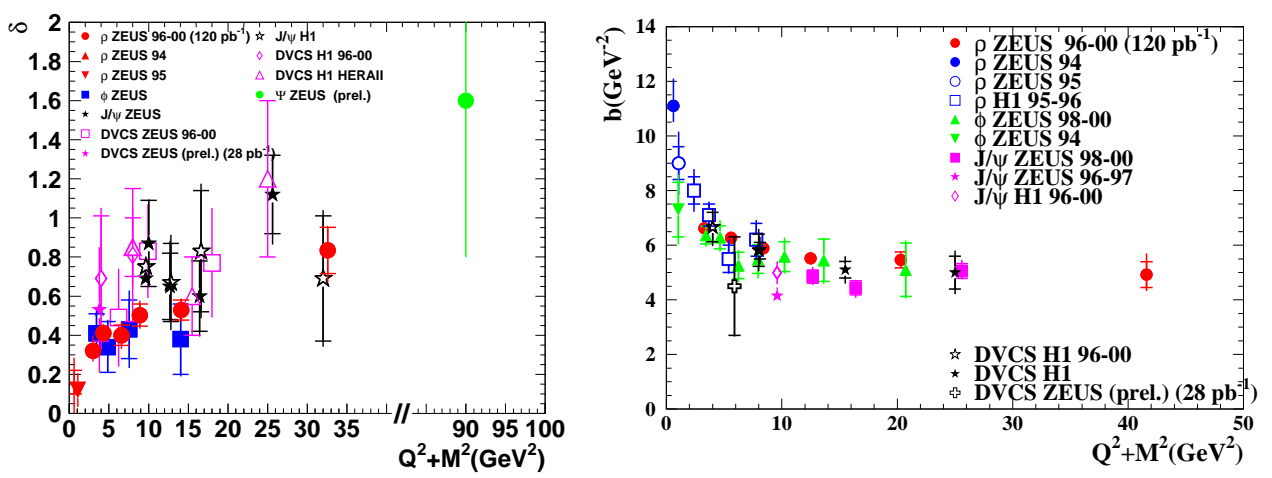

Fig. 2. Left: power $\delta$ as a function of $Q^{2}+M_{V M}^{2}$ for various VMs and DVCS. Right: slope $b$ as a function of $Q^{2}+M_{V M}^{2}$ for various VMs and DVCS. See text for details.

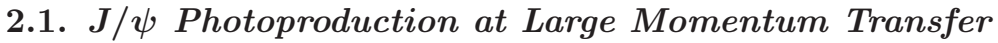

The ZEUS Collaboration studied recently ${ }^{4}$ the process of proton-dissociative photoproduction of the $J / \psi$ meson: $\gamma p \rightarrow J / \psi Y$, which dominates at larger momentum transfer $|t|>1 \mathrm{GeV}^{2}$ (proton dissociates into low-mass nucleon state $Y$ ). This is a two-scale process with large $J / \psi$ mass and momentum transfer $t$ and in the leading logarithmic (LL) QCD approximation it can be described by the exchange of a gluon ladder. At large $|t|$ values, the cross section is expected to decrease according to power law (in contrast to "elastic" VM production, where exponential $t$-dependence is observed). At large energy $W$ this process should be sensitive to Balitsky, Fadin, Kuraev, Lipatov (BFKL) dynamics of the gluon ladder (unordered gluon momenta) resulting in strong energy dependence of the cross section, in contrast to weak energy dependence typical for Dokshitzer, Gribov, Lipatov, Altarelli, Parisi (DGLAP) evolution (ordered momenta). As can be seen in Fig. 3, at large $|t|$ the differential cross section decreases according to power law and the cross section rises with increasing energy $W$. This rise is not reproduced by the EMP model (DGLAP dynamics) and is overestimated by the GLMN model (BFKL dynamics) and FSZ model (relating rise of the cross section to gluon density in the proton). In this situation no clear evidence for BFKL dynamics can be claimed.

\section{Inclusive Meson Production}

\subsection{Inclusive Photoproduction of Light Mesons}

The H1 Collaboration published recently ${ }^{5}$ results on inclusive photoproduction of the $\rho, K^{* 0}$ and $\phi$ mesons. All of them were detected through their decays into two charged hadrons. Inclusive transverse momentum and laboratory rapidity distributions were measured: as can be seen in Fig. 4 the former ones fall according to power law with the same average transverse kinetic energy for all mesons and the latter are pretty flat within the detector acceptance. The same average transverse kinetic 
ZEUS

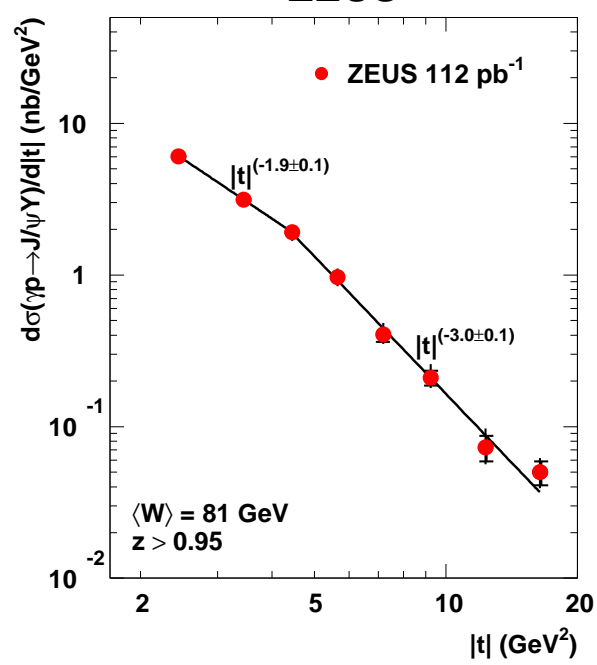

ZEUS

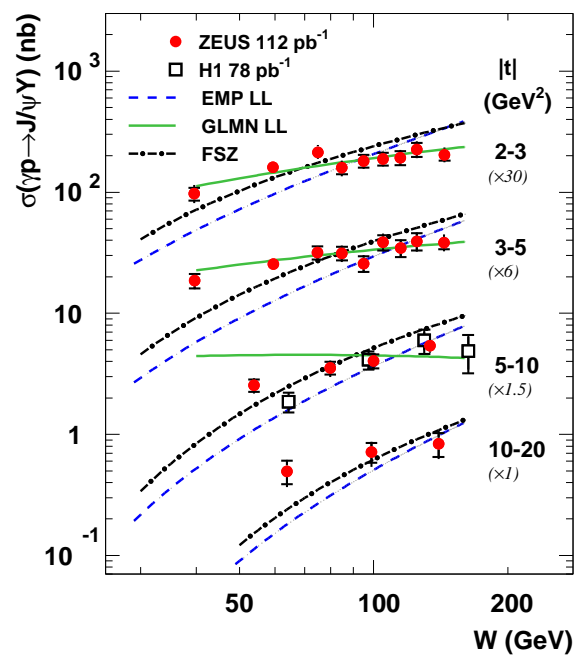

Fig. 3. Left: the $|t|$ dependence of the differential cross sections for the process $\gamma p \rightarrow J / \psi Y$ (the solid lines are the results of power fits). Right: the $W$ dependence for this process in four $|t|$ bins. Also the $\mathrm{H} 1$ data are plotted for comparison. The lines show the predictions of several calculations mentioned in the text.

energy for all mesons is consistent with thermodynamical picture of the hadronisation process. Two phenomenological models (PHOJET10 and PYTHIA6.2) containing "soft" fragmentation of partons were unable to reproduce the experimental data.

\subsection{Heavy Flavoured Meson Electroproduction}

The production of the charmed mesons $D^{+-}$and $D^{0}$ in DIS has been measured by the ZEUS Collaboration. ${ }^{6}$ The charged $D$ mesons were detected through decays into three charged hadrons and the neutral ones - into two charged hadrons. The ZEUS microvertex detector was used to reconstruct the displaced secondary vertices which reduced the combinatorial background to the $D$ meson signals. The charmed mesons are produced predominantly via photon-gluon fusion which makes this process quite sensitive to gluon density in the proton (Parton Density Functions - PDFs). There are also three "hard" scales in it: virtuality $Q^{2}$, mass of the charmed quark and transverse momentum of the $D$ meson so one can expect the perturbative QCD to work here. To verify this, measured cross sections were compared with the predictions of the next-to-leading-order QCD (model HVQDIS) using proton PDFs extracted from inclusive DIS data. A good description of the experimental results was found: selected comparisons are shown in Fig. 5. 

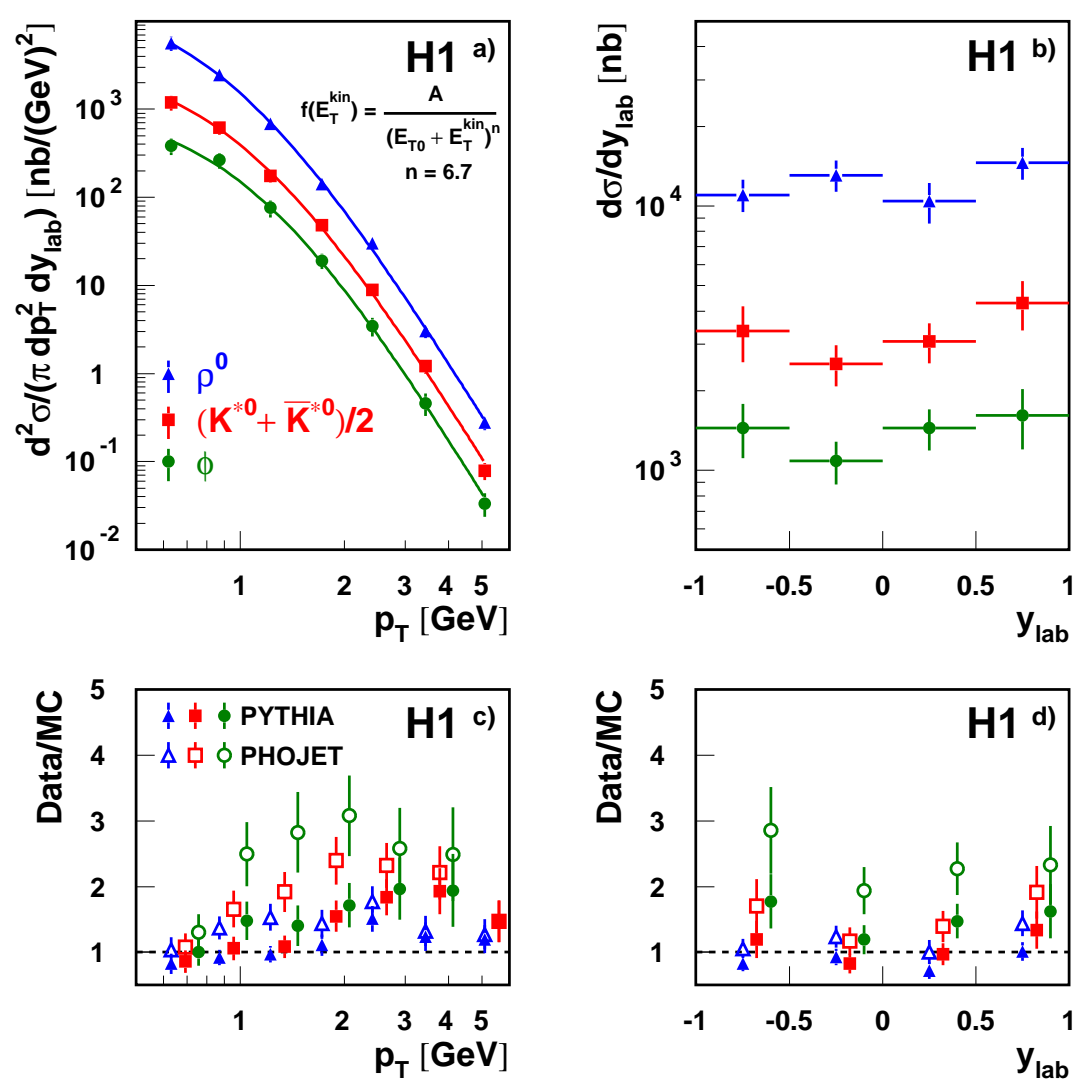

Fig. 4. The differential cross sections for $\rho, K^{* 0}$ and $\phi$ mesons as a function of transverse momentum (a) and rapidity (b). The curves in Fig. (a) correspond to the power law with $n=6.7$. The ratios of data to Monte Carlo predictions are also shown (c,d).

\section{Summary}

HERA collider was a meson factory: from wealth of recent results only selected ones were presented above. Here several conclusions are listed:

- Transition between soft and hard hadronic interactions is observed in diffractive vector meson production; pQCD provides semi-quantitative description of the hard interactions,

- Inclusive light meson production is still a real challenge for soft fragmentation phenomenology,

- Perturbative QCD is quite succsessful in description of heavy flavour meson production. 

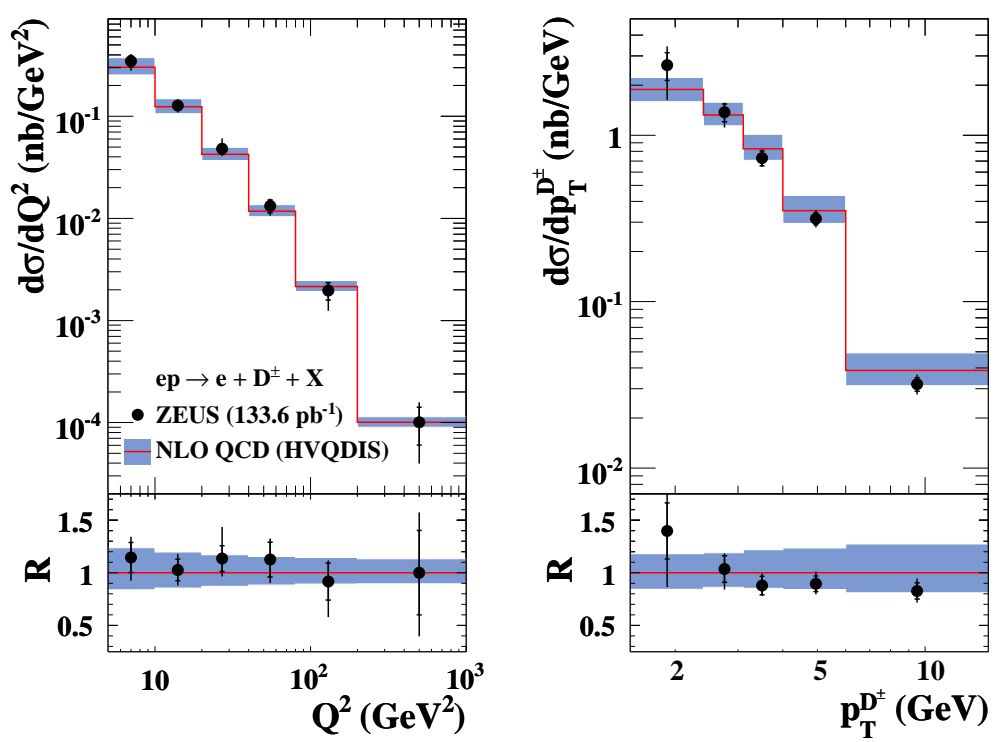

Fig. 5. Differential cross sections for $D^{+-}$mesons in function of $Q^{2}$ (left) and $p_{T}^{D}$ (right) compared to NLO QCD predictions of HVQDIS. The shaded regions represent uncertainties of the model. The ratios, $R$, of the data to HVQDIS prediction are shown in lower sections of the plots.

\section{Acknowledgments}

This work is supported by the Polish Ministry of Science and Higher Education, project No. DPN/N188/DESY/2009.

\section{References}

1. S. Chekanov et al. [ZEUS Collaboration], PMC Physics A1, 6 (2008).

2. F. D. Aaron et al. [H1 Collaboration], Diffractive Electroproduction of rho and phi Mesons at HERA, preprint DESY 09-093.

3. S. Chekanov et al. [ZEUS Collaboration], Phys. Lett. B 680, 4 (2009).

4. S. Chekanov et al. [ZEUS Collaboration], JHEP 05, 085 (2010).

5. F. D. Aaron et al. [H1 Collaboration], Phys. Lett. B 673, 119 (2009).

6. S. Chekanov et al. [ZEUS Collaboration], Eur. Phys. J. C 63, 171 (2009). 